\title{
Spin-Orbit Torque Driven Multi-State Device for
}

\section{Memory Applications}

\author{
Selma Amara, Ulan Myrzakhan, Abdulmohsen Alsaui, Meshal Alawein, \\ and Hossein Fariborzi \\ CEMSE Division, King Abdullah University of Science and Technology (KAUST) \\ hossein.fariborzi@kaust.edu.sa
}

\begin{abstract}
We report the design, fabrication and measurement of a spin-orbit torque (SOT) driven multi-state memory. The multi-state memory is based on a $3-\mathrm{nm}$ thick $\mathrm{Ni}_{81} \mathrm{Fe}_{19}$ (Py) patterned in the shape of four intersecting ellipses (octagram). Writing is performed by injecting a charge current along one of the Pt wires, which generates a transverse pure spin current capable of switching Py between its states. Information is read through a magnetic tunnel junction (MTJ) using four-point technique.

(Keywords: Spintronics, multi-state memory, SOT and MTJ)
\end{abstract}

\section{Introduction}

Over the last decades, complementary metal-oxidesemiconductor (CMOS) technology has been the predominant computing technology. However, CMOS technology has been facing many challenges such as increased power density and degraded energy efficiency due to various quantum and leakage effects arising in deeply scaled devices. These issues have triggered extensive research efforts on many "beyond-CMOS" technologies, some of which are not based on charge as a state-variable. A promising candidate is spintronics, a technology in which the electron spin, in addition to its charge, is used as a state-variable for storage and computation [1].

Magnetic tunnel junctions (MTJs) are the main spintronic-based storage and sensing devices, and are the basis of today's magnetic random-access memories (MRAMs). MTJs offer a number of attractive features such as cyclability, nonvolatility, high switching speeds, high scalability, low writing power and compatibility with CMOS technology. A simple MTJ consists of two ferromagnetic layers sandwiching an insulating nonmagnetic layer, and it works on the principle of tunnel magnetoresistance (TMR) which converts a magnetic signal into an electrical signal. The magnetic information is written in a thin ferromagnetic layer called the "free layer" either by current-generated magnetic fields, spintransfer torques (STTs), or more recently, by spinorbit torques (SOTs), which brings several advantages over the former methods. The written information is evaluated by comparing it to the magnetization of a thick or antiferromagneticallypinned ferromagnetic "reference layer" [2]. The stored information can then be electrically read by measuring the resistance of the MTJ which depends on the relative alignment of the magnetizations of the two ferromagnetic layers. Typically, the ferromagnets are ellipses having two easy directions (resulting from shape anisotropy), and thus provide two resistance values denoted as "parallel" and "antiparallel", effectively representing one bit of information ("0" and "1").

Although MRAM is believed to be a promising candidate for a universal memory, at the moment it is an expensive technology. MRAMs are limited by the compromise between bits density and information reliability. The reason is that each cell is capable of storing only one bit of information. An increase in memory capacity can only be accomplished by reducing the cell size, while maintaining a strong anisotropy barrier in face of thermal fluctuations to avoid erroneous switching. To overcome this problem, one can alternatively construct a multi-state magnetic cell (e.g., by shape engineering the ferromagnets) to introduce multiple magnetic easy directions $(>2)$. Recently, such concept has been used to demonstrate four- and six-state spintronic memories that utilized the configurational anisotropy of square and triangular $\mathrm{Ni}_{81} \mathrm{Fe}_{19}$ (Py) elements, respectively [3]. Similarly, [4] demonstrated six stable magnetization states in a Py film shaped as three intersecting ellipses. The latter work also suggested that such intersecting ellipses can be used as the free layer in an MTJ to obtain six states in a single memory cell. In this work, we report the design, fabrication and measurement of a multi-state memory cell based on SOT driven four intersecting ellipses (octagram).

\section{Proposed Memory Cell}

The proposed multi-state memory cell is based on an MTJ with four intersecting Py films of elliptical shape (both in its free and reference layers) centered at the same point, but rotated by $45^{\circ}$ from one another. The OOMMF [5] micromagnetic simulations, illustrated in Fig. 1, show the existence of eight stable magnetization states in the overlapping region of the 
proposed structure. In this work, we pin the reference layer along the $y$-axis $\left(\theta=0^{\circ}\right.$ in Fig. 1). The resistance of the MTJ is calculated using the following equation [4]

$$
\mathrm{R}_{\beta}=\mathrm{R}_{\mathrm{AV}}-1 / 2 \Delta \mathrm{R} \cos (\beta)
$$

where $R_{A V}=1 / 2\left(R_{A P}+R_{P}\right)$ and $\Delta R=R_{A P}-R_{P}$. Here $R_{P}$ and $R_{A P}$ are the resistances in the parallel and antiparallel configurations, respectively, and $\beta$ is the angle between the magnetizations of the free and reference layers. Using (1), eight resistance states would be expected: $\mathrm{R}_{0^{\circ}}, \mathrm{R}_{45^{\circ}}, \mathrm{R}_{90^{\circ}}, \mathrm{R}_{135^{\circ}}, \mathrm{R}_{180^{\circ}}, \mathrm{R}_{225^{\circ}}$, $R_{270^{\circ}}$ and $R_{315^{\circ}}$. However, the symmetry of the system leads to degenerate resistance states, namely $\mathrm{R}_{45^{\circ}}=$ $\mathrm{R}_{315^{\circ}}, \mathrm{R}_{90^{\circ}}=\mathrm{R}_{270^{\circ}}, \mathrm{R}_{135^{\circ}}=\mathrm{R}_{225^{\circ}}$. This effectively generates five distinct resistance states (instead of eight). The degeneracy can be removed, one should break the symmetry of the system. For example, this can be done by tilting the reference layer with respect to the free layer to obtain eight distinct resistance states for the MTJ, the latter of which can then be used as the core of an eight-state memory device.

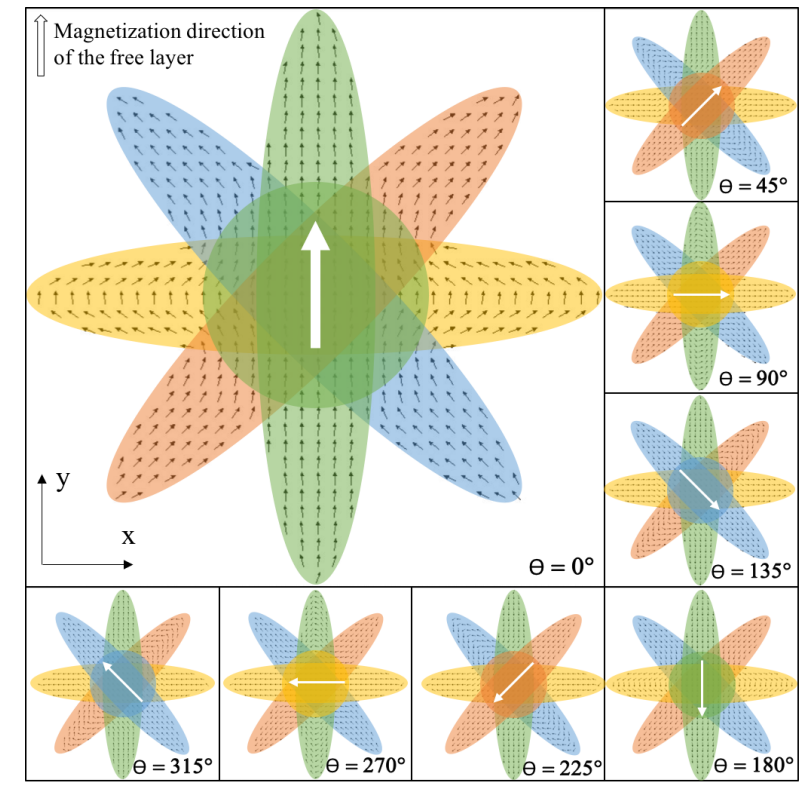

Fig. 1: OOMMF micromagnetic simulations showing the existence of the eight stable magnetic states in the overlapping region of four intersecting elliptical Py films. The magnetization is represented using a white arrow within each element, and is characterized by an angle $\theta$ (measured clockwise with respect to the y-axis).

\section{Fabrication}

The MTJ multilayer stack we fabricated consists of: $\mathrm{Py}(3 \mathrm{~nm}) / \mathrm{MgO}(1.5 \mathrm{~nm}) / \mathrm{Py}(20 \mathrm{~nm}) / \mathrm{Ru}(3 \mathrm{~nm})$. This is illustrated in Fig. 2. The multilayer stack was deposited using a magnetron sputtering system and patterned with standard photolithography. Finally, $\operatorname{Ti}(10 \mathrm{~nm}) / \mathrm{Pt}(350 \mathrm{~nm})$ contacts are deposited to electrically control the device.

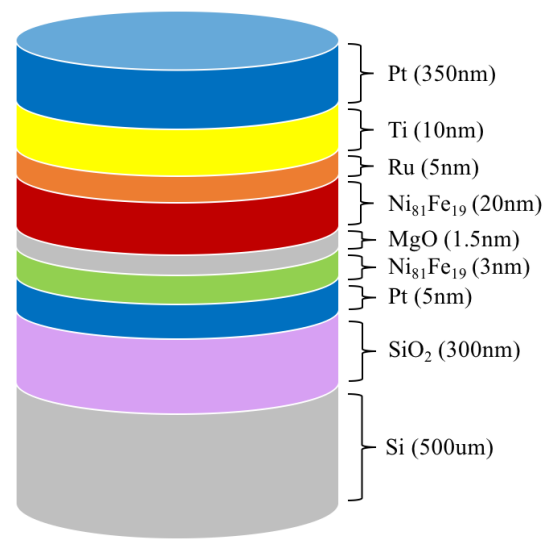

Fig. 2: Cross-section view of the fabricated multilayer stack.

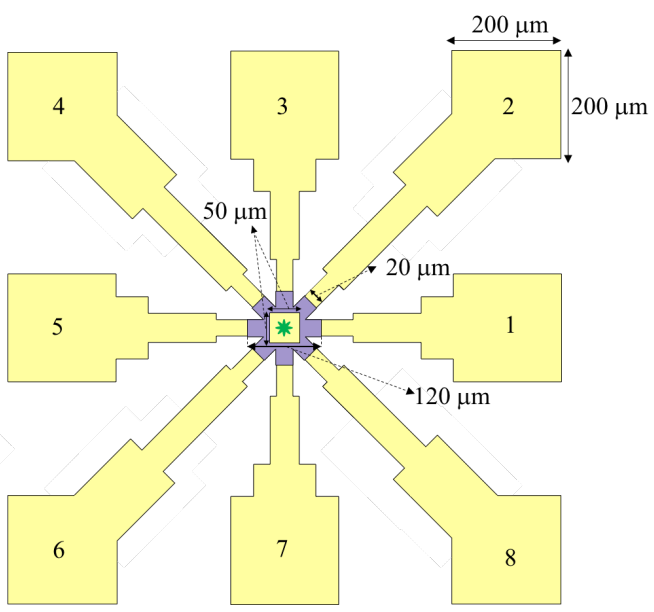

(a)

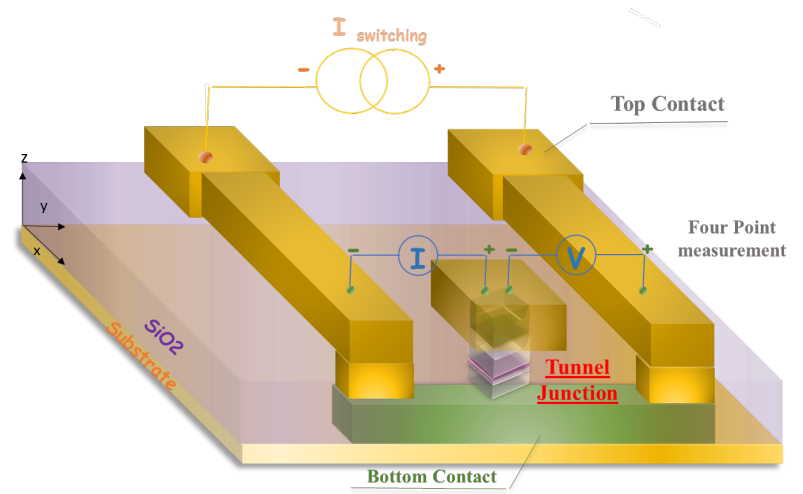

(b)

Fig. 3: (a) Top view of the layout with the pad numbering and the MTJ stack location. (b) Four-point measurement.

SOT is used to switch the free Py layer between its stable magnetization states. To accomplish this, the MTJ is centered at the intersection region of four 
Pt wires, with each wire coinciding with the major axis of an ellipse (see Fig. 3(a)). Injection of an inplane charge current in any Pt wire (Fig. 3(b)) scatters electrons of opposite spin in opposite directions as a result of the spin Hall effect (SHE). This in turn generates an accumulation of electrons of opposite spin at the boundaries of the wire, which triggers a spin diffusion process. Effectively, this diffusion injects a transverse pure spin current that can then switch the magnetization of the free Py layer by spin angular momentum transfer. Fig. 4 shows an SEM image of a fabricated sample.

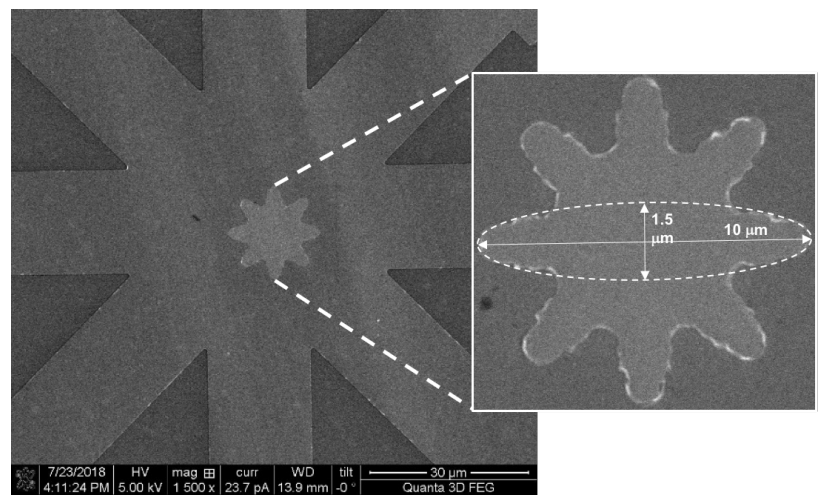

Fig. 4: SEM image of the fabricated device.

Characterization and Results

For the characterization of the fabricated device, one probe was fixed at the top contact of MTJ for reading and two probes were placed on the side pads for writing the current in the desired direction (Fig. 3(a)). For example, $\mathrm{R}_{0^{\circ}}$ was measured by driving the writing current from pad 5 to pad 1 and reading the resistance in four-point measurement mode (Fig. 3(b)) between the top contact of the MTJ and pads 3 and 7. The writing pads were changed for every write operation. The device was characterized using a writing current of $10 \mathrm{~mA}$ and a reading current of $1 \mathrm{uA}$. Measurement results are illustrated in Fig. 5. As expected, the device possesses five resistance states: $R_{0^{\circ}}, R_{45^{\circ}}=$ $\mathrm{R}_{315^{\circ}}, \mathrm{R}_{90^{\circ}}=\mathrm{R}_{270^{\circ}}, \mathrm{R}_{135^{\circ}}=\mathrm{R}_{225^{\circ}}, \mathrm{R}_{180^{\circ}}$. To better assess the device performance, a plot of Eq. (1) (blue curve in Fig. (5)) was generated for all the possible angles e.g. relative alignment of the magnetizations in the free and reference layers. As can be seen the measured resistance states are in close agreement and follow the trend predicted by equation.

\section{Conclusion}

In this work, we demonstrated a magnetic structure for implementation of multi-state memory applications based on SOT switching. We observed

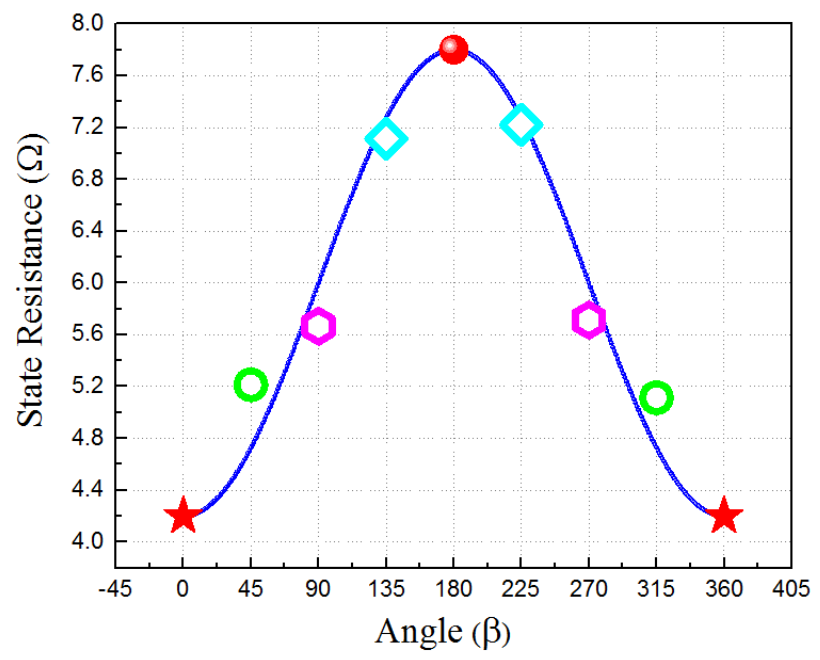

Fig. 5: Resistive states of the proposed memory cell.

five different resistive states as expected. By breaking the symmetry between the easy axes of the free and reference layers (e.g., by pinning the reference layer in the direction other than, and not right between, the easy axis direction of the ellipses), the exactly same proposed memory cell can be designed to provide all eight resistive states and enable the storage of three bits of information.

\section{References}

[1] Wolf, S. A., D. D. Awschalom, R. A. Buhrman, J. M. Daughton, S. Von Molnar, M. L. Roukes, A. Yu Chtchelkanova, and D. M. Treger. "Spintronics: a spin-based electronics vision for the future." Science 294, no. 5546 (2001): 1488-1495.

[2] Zeinali, Behzad, Jens K. Madsen, Praveen Raghavan, and Farshad Moradi. "Ultra-Fast SOTMRAM Cell with STT Current for Deterministic Switching." In Computer Design (ICCD), 2017 IEEE International Conference on, pp. 463-468. IEEE, 2017.

[3] S. Wasef, S. Amara, M. Alawein, H. Fariborzi," Multibit Memory Cells Based on Spin-Orbit Torque Driven Magnetization Switching of Nanomagnets with Configurational Anisotropy," IEEE EDTM-2018 Kobe, Japan, 2018.

[4] Telepinsky, Yevgeniy, Vladislav Mor, Moty Schultz, Yu-Ming Hung, Andrew D. Kent, and Lior Klein. "Towards a six-state magnetic memory element." Applied Physics Letters 108, no. 18 (2016): 182401.

[5] M. J. Donahue and D. G. Porter, OOMMF User's Guide, Version 1.0, Inter- agency Report NISTIR 6376, Gaithersburg, MD, September 2017. 